\title{
Optical Waveguides and Terahertz Signal by Finite Element Method: A Survey
}

\author{
T. V. Smitha ${ }^{1}$, Madhura $\mathrm{S}^{2}$, Shreya $\mathrm{N}^{2}$ and Sahana Udupa ${ }^{3}$ \\ ${ }^{1}$ Department of Mathematics, RV Institute of Technology and Management \\ Bangalore. \\ ${ }^{2}$ Department of ECE, RV Institute of Technology and Management \\ Bangalore. \\ ${ }^{3}$ Department of ISE, RV Institute of Technology and Management \\ Bangalore. \\ smithatv.rvitm@rvei.edu.in, Madhu4tulip@gmail.com, \\ shreyan_ec19.rvitm@rvei.edu.in, sahanaudupasv_ec19.rvitm@rvei.edu.in
}

\begin{abstract}
This paper examines the use of the Finite Element Method (FEM) in the field of optical waveguides and terahertz signals, with the main goal of explaining how this method aids in recent advances in this field. The basics of FEM are briefly reviewed, and the technique's application to waveguide discontinuity analysis is observed. Second-order and higherorder derivatives result from optical waveguide modeling, which is significant for information exchange and many other nonlinear phenomena. The use of FEM in the improvised design of hexagonal sort air hole porous core microstructure fibers, which produces hexagonal structure cladding and rectangular-shaped air holes in the fiber core for excellent terahertz signal transmission, was also observed. These modifications were intended to improve the fiber's properties in comparison to other structures. This approach verifies that the fiber has high birefringence, low material loss, a high-power fraction, and minimal dispersion variation. The features of square-type microstructure fiber are investigated. A folded-shaped porous cladding design is recognized for sensing applications. This type of photonic crystal fiber is also known as FPPCF since it features circular air holes. The most approximate findings of this application are obtained using FEM. In comparison to many other approaches for various applications, it is evident that FEM is a powerful and numerically efficient tool. This work does a survey of optical waveguides and terahertz signals using the Finite Element Method. Terahertz signals can be used in conjunction with electromagnetic waves to identify viruses. Thus, Terahertz signals are employed in real-world applications such as fuel adulteration, liquid metal synthesis, and virus detection.
\end{abstract}

Keywords: Finite Element Method, Terahertz, Optical waveguides, Microstructure Fiber Management Introduction, Photonic Crystal Fiber, Petrol adulteration, optical modes, liquid metal generation 


\section{Introduction}

The optical waves are the main components of optical circuits that can control the couple, switch, multiplex, and de-multiplex signal. Optical Waves use various diffusion approaches, such as the beam propagation method that works well for bend guides and also provides comparable results, finite difference time domain for the ability to handle complication in geometrical ease, finite element analysis, and the most effective method for achieving and estimating boundary value problem results. It is capable of handling complex geometry. Frequency and wave can be resolved with many other methods including beam propagation or using the time domain and other modes with the aid of the FEM. The Polarization field element is found in electromagnetic waves with FEM and optical mode features. Most optical waves are tightly packed for transmitting nanometer distance signals within. Waveguide Bend, Photonic Bandgap structures, Multi-mode interference are optical waveguides. The structural geometry acts as an important factor because of the low refraction region. The field of optical waveguides has seen a drastic advancement over the years. Some of these advances include the cases of them being non-homogeneous and non-linear. In such cases, it goes from being difficult to almost impossible to attain theoretical solutions. Hence, the computational methods are used to get approximate solutions with high performance. FEM is a widely used numerical technique in the field of engineering mathematics and hence, it is one of the proficient tools to carry out guided wave problems. The fundamentals of FEM like Theoretical framework, nodal and edge elements, and Perfectly Matched Layer (PML) are introduced. Further, the FEM technique is described in waveguide discontinuity analysis [1-4].

Terahertz radiation is an electromagnetic wave whose frequency ranges from $0.3 \mathrm{THz}$ to $30 \mathrm{THz}$ and has a wavelength of radiation ranging from $0.1 \mathrm{~mm}$ to $1 \mathrm{~mm}$. It has a wide range of applications starting from the biomedical field like cancer diagnosis through imaging. It also finds application in spectroscopy and communication. The guided medium of Terahertz radiation in the optical waveguide is hindered by various factors like degradation of property of material and complications from other devices. Hence, there is a need to manipulate the design of the waveguide. This is where microstructure fiber comes into the picture. They are optical fiber waveguides in which the transmission of radiation is acquired by altering the structure of the waveguide. This provides better guiding characteristics. Like said before, the material of the fiber plays a key role in this process. Usage of porous core fiber is more effective than solid core fiber because it provides low material loss and low dispersion variation. To further ensure the effective guidance of the radiation we obtain higher birefringence by making the cladding region asymmetrical. Several authors who have worked in this area have produced results 
proving to get the desired characteristics of high birefringence, reduced effective loss, and low dispersion [5-8].

Here, we aim to improve the characteristics of the guided wave by modifying the design. In this paper, the cladding region of the fiber is a hexagonal type structure. The air holes are also hexagonal-shaped. This is done to increase the quantity of the air holes and decrease the bulk material in the waveguide. To further improve the characteristics, the core region of the fiber is introduced with rectangular-shaped air holes. This provides higher birefringence. To confirm these results numerically, FEM computation is used [9]. These designing improvements are highly advantageous in the field of terahertz application. Another type of structure called square type structure is introduced further to analyze the parameters of the microstructure fiber. The results obtained are numerically confirmed by the FEM numerical analysis [2831].

Photonic Crystal Fiber (PCF) comes under the optical waveguides and is known to have greater characteristics compared to other waveguides. PCF has air holes in its cladding region which leads to increased effective material loss (EML). In PCF the light-guiding mechanism used is Total Internal Reflection. To decrease the EML a porous core photonic crystal fiber (PC-PCF) is introduced where the core region also has air holes along with the cladding region. This reduces the EML. The guiding mechanism for PC-PCF is the photonic bandgap. The guiding mechanism also depends on the refractive index of the material. The application of PCF as sensors has been an area of research for quite a long time. However, recent times have seen an extensive increase in this topic. Recent developments in this area are the usage of PCF as temperature sensors, humidity sensors, pressure sensors, etc. In this paper, the PCF characteristics are modified to receive the best sensing application. Further, these characteristics are numerically analyzed by my numerical analysis mesh generation method using FEM. It provides high accuracy by providing the most approximate solution. Properties like effective area, nonlinearity, and confinement loss are also investigated.

Most methods of doing the work on terahertz waveguide are difficult because of variation of the material import for the given frequency. Because of the low refractive index, opaque materials are used for the study of optical waveguides. This is the main disadvantage for the method because if we use another type of materials like polymers, glasses there is power dissipation [10]. By the experiment, we can get the radially symmetric photoconductive antenna by FEM. Most of the terahertz polarized waves does not hold good for overlapped for polarized radially guided wave. Because of the use of the terahertz method, we can easily calculate the wave propagation through air symmetrically geometrically shaped bodies. For example, pipes, 
brass, metals, etc. These changes their shape for displacement, velocity derivatives [8]. Linear waves cannot be used for coupling the fire inputs. But by using FEM guides the wavelength and efficiency, as approximations are high in this data.

Terahertz spectroscopy system can be used in the detection of influenza virus forming patterns of spectrum sensing chips are introduced and by FEM or Finite Time-Domain Method (FDTD) can be implemented analyzed and later can be simulation can be done. This method uses a nano antenna to detect biomaterial. A sample will be collected and high accuracy can be obtained. Terahertz signal can be used in designing liquid metal components as it has high electric control. By this, we can design terahertz devices.

Photonic crystal fiber is a new alternative that can be used in petrol adulteration as a degree of freedom, design, flexibility, high adulteration, etc. Electromagnetic spectrum can be used and propagation media could be in terahertz signal. Zeonex material can be used and fuel can be adulterated. Oil refinement can be done and proposed using 2D hollow code cladding

\section{Fundamentals of finite element method and its techniques being applied in waveguide discontinuity analysis}

The theoretical framework of FEM: Under FEM, instead of solving the region as a whole, the area is divided into a finite number of elements to which principle variations are applied. Each element is considered as a discretized model and they are solved individually. Further, all the elements are combined back into their original form. The elements can be of various shapes and depending on the shapes uniform or non-uniform mesh are used. Due to this factor, FEM works well for complex geometry and hence has vast applications. It is a highly successful method because it can also be used with non-homogenous and non-linear problems. Apart from the variational method in FEM, the other method is a weighted residual method. In this method, the error from the acquired solution is considered as the residue. It can be applied to optical waveguides with leak where variational methods cannot be used [2].

Nodal and edge elements: The different types of elements in FEM for 2D or 3D problems could be triangular and tetrahedral elements that are used respectively in general. As the complexity of the problem increases the order of the polynomial can also be increased to obtain better results. The lowest order elements are based on vector basis function. The constant tangential and linear normal basis functions, in particular. For one of the edges, the tangential component is constant and for the rest of the edges it is zero, and the normal component is 
linear all along [1]. For higher-order components, linear tangential and quadratic normal vector basis functions are used.

Perfectly Matched Layer: The utility of suitable absorbing boundary conditions is important in optical waveguide devices. This is to reduce the spurious solutions occurring from reflections through edges. This is where PML comes into the picture. The recently developed PML does not allow the field to split and hence has been used widely in FEM. The PML parameter such as its permittivity and permeability is observed to control the tempering of the electromagnetic field in PML regions. However, PML has certain limitations. They are only completely reflectionless for exact continuous-wave equations. PML also requires that the medium be invariant to support the acquiring solutions.

Beam propagation method (BPM): It is a vastly used Method for light propagation. It shows the study of variations in the longitude of waveguides. FEM is applied to BPM (FE-BPM) where the transverse part of the pane is discretized. This is one of the places where non-uniform mesh generation is applied. This increases the accuracy of the solution without tampering with the efficiency of propagation. The domain boundary conditions have always been a limitation in this method. This however can be rectified with the help of anisotropic PM [3]. This can be implemented on both 2D and 3D structures. BPM only takes forward reflection waves and doesn't consider backward reflection.

Optical mode: Optical modes mainly depends on the electromagnetic wave and also depend upon polarization. It mainly consists of electric field and quasi modes by solving in vector analysis 2-dimensional optical waveguides can be analyzed by optical modes ineffective areas like a high refractive index. The FEM solver can solve these modes. It is one of the easiest methods for solving optical modes. Designing and analysis may design multiplexer, coupled with an optical waveguide. Beam propagation method analysis cannot be used as it has confinement loss and losses due to bending of light. Polarization mode is increased by bending of light and these can be solved in the Maxwell equation. The different refractive indexes can be combined and computed using FEM and they can be displayed in an optic waveguide mode. Second-order and higher-order parameter gives higher index value.

The optical waveguide is not a practical solving technique but FEM analysis of electromagnetic waves is possible. A practical waveguide is easy to solve but theoretically, it's a very difficult part of the analysis. But a recent development in computer programming it is possible to solve and analyze FEM describes optics waveguide fields in dual ends of the wire. 
Optical waveguide gives zero dispersion point, birefringence comparison makes easier for choice for optical waveguide. Small and minute optical devices are designed by this optical device hence large diameters are needed for confinement loss, transmission loss. Many algorithms are used for confinement computation like the FDTD algorithm and FEM and this can be solved by using the Eigen vector. Multimode interface with a difference of pi for all the images in image processing of optical waveguide. Various mirrored images and multi images are formed in this condition

Waveguide discontinuity analysis: It has two domains namely the Frequency domain and time domain.

Frequency domain (FD-FEM): waveguide discontinuity analysis takes into account both forward and backward reflections in the discontinuity region. The frequency-domain has largely been used to solve FEM problems. It is developed by using anisotropic PML absorbers to avoid mode expansion to be used in $2 \mathrm{D}$ and 3D waveguide discontinuity problems. Triangular nodal element and tetrahedral edge element are used respectively. One of the 2D-FDFEM is the two-mode multiplexer (TM-MUX). It has the property of not disturbing the wavelength phase shifter for proper transmission purposes and multimode interference waveguides. The structure of the waveguide is butterfly-shaped and it includes a 3-dB coupler. PML is used to prevent reflections in multimode interference waveguides. Before the use of PML in TMMUX it uses to have input/output ports. By altering these ports, the two-mode multiplexer could be converted to a mode de-multiplexer.

Time-domain: BPM's are usually not used in waveguide discontinuity analysis due to their one-sided reflectivity [4]. However, if the frequency of modulation is lower than the frequency of career, then the domain can be divided into elements for the time domain. An algorithm called TD-BPM has been developed for this consideration. They can solve problems with waveguides and have an amplified PML that is efficient. Several characteristics like wavelength are analyzed using this method. Taking an application in couplers with dielectric pillars, a $90^{\circ}$ is taken on the square array and the lattice constant of $0.580 \mu \mathrm{m}$ is given. The following results were observed, with a radius of $0.104 \mu \mathrm{m}$ this structure gave out almost $100 \%$ transmission. 
Numerical analysis for channel waveguide: FEM is one of the prominent methods in optical waveguide like a dimensional waveguide, linear waveguide, slab waveguide, oscillators, etc. .and these can be solved in by mesh generation it can be neither TE nor TM and it can be solved by first-order modes. We can use the finite difference method with mesh refinement with division. It is used to represent waveguide for different refractive index and it is diffused it very easily. FEM uses different methods to find solutions for one-dimensional, two-dimensional, and three-dimensional functions. We can use the shape functions domain with discrete values and other nodes and versatile methods to solve for the optical waveguide is FEM.

\section{Finite element analysis of optical waveguide}

Mostly in FEM, the field area is divided as triangular, rectangular, or curvilinear finite elements. FEM accepts discontinuity of refractive index. FEM vectorial analysis is very needed for strong hybrid modes, polarization, switching and it can be implemented by Maxwell's equation in XY plane and del operator. The generalized Eigenvalue equation by Maxwell equation is given by:

$$
[\mathrm{K}](\varphi)-\beta 2[\mathrm{M}](\varphi)=(0)[13]
$$

FEM can handle complicated geometries with ease as it has symmetric properties. It can solve high-order equations. The vectorial analysis could also be done for hybrid modes by the Helmholtz wave equation [11].

Shape functions are obtained by converting continuous field functions to discrete values with the total number of nodes. It is continuous across the adjacent triangle and it can be viewed by surface with many faces.

Solving waveguide problems by FEM is easy for storage requirements and computational efforts. By sparse matrix for more accurate solutions but the disadvantage with high density with the algorithm. This matrix solution technique does not need any initial guess for the field Eigenvector, which is generated from random members to avoid any initial bias [12].

We have a simple phase index channel waveguide, for example, a simple mesh of 6 and $7 \mathrm{x}$ side and $\mathrm{y}$ side mesh of half-plane $\mathrm{x}>=0$. Half the cross-section of the waveguide is defined by 84 first-order elements and a single-phase index channel waveguide with some infinite element. Modes of channel waveguide are neither pure TE nor pure TM. It is classified by transverse $\mathrm{x}$ direction and $\mathrm{y}$ direction with lower mode $\mathrm{m}$ and $\mathrm{n}$ as quasi TM modes. Effect of mesh analysis mesh refinement and accuracy improves by mesh division. It can be noticed by high 
order mode converges slower than fundamental modes with more mesh division to represent faster spatial field variation.

Another example for calculating the approach with the most correct values after examining is mesh engine analysis. By applying the mesh engine technique core cradle densities with high or least is generated. Mostly because of proximate results. Most partial derivatives and other calculations and approximations could be done. By creating an algorithm using data and application, one can take any symmetrical geometrical figure for examples like a triangular shape and record or locate points and connections by joining the points which are created in the triangles $(i, j)-(i+1, j)-(i, j+1)$ and $(i+1, j)-(i+1, j+1)-(i, j+1)$. Connections are done from a corner from the top to the left corner to the bottom right corner. Pointing Straight for joining the first order triangle. If points are done in clockwise directions nodes are done in an anticlockwise direction. By points and nodes, we can produce a 2-dimensional array. The first array holds the mode date, where node values and refractive index are stored and connected. The second arrays store data of given signals [20-22].

The dark region represents the border of the given elements, if we want to increase the strength changes the values of $\mathrm{x}$ and $\mathrm{y}$ will give better performance Meshing engines are directly proportional to several elements but it gives disadvantage also because of more complications, it is not important to do high meshing calculation at all parts of the examining area, but mainly at the core of the waveguide.

Area meshing is the calculation of areas with different densities and complexity in the core. But if we change the values of the $\mathrm{X}$-axis it depends on the node and elements, even the geometry can change for variations. So, changes should be done in both the $\mathrm{X}$ and $\mathrm{Y}$-axis. The main advantage of the above example is time-consuming because of symmetric waveguide half-waves guide gives the answer and the result of the other could be found easily because of the symmetric property.

Another area where FEM could be used is in the Graphically user interface (GUI). The user can access it easily and it has a user-friendly environment. We can produce values of field distributions by a graphical user interface. Students can use the FEM model solver and experiment on optical waveguides for no cost. Complexity reduces using Graphic user interface, user can even do higher-order own design, implementation, and configuration of problem. 
J. Sustain. Wireless Syst., vol. 03, no. 2, pp. 68-86

https://doi.org/10.36548/jsws.2021.2.002

\section{DESIGN AND ANALYSIS OF MICROSTRUCTURE FIBER}

\subsection{HEXAGONAL POROUS CORE MICROSTRUCTURE FIBER}

The design proposed is a unique structure designed to get the desired characteristics of the optical waveguide. The design consists of a hexagonal array of air holes in the cladding region and the air holes in the core are rectangular shaped. The hexagonal array creates a hexagonal lattice which gives better light captivity. The rectangular shape provides asymmetry in the polarization mode which increases the birefringence. The air filling fraction is taken as 0.95, if taken more the air holes overlap and cause a fabrication problem. The length of each arm of hexagonal cladding is taken uniformly as $\mathrm{r}$. The length and width of the core are $5 \mathrm{r}$ and $4.3 \mathrm{r}$ respectively. The rectangular air holes have different heights due to their asymmetry. Further, a circular Perfectly Matched Layer (PML) is introduced in the outer region of cladding. A $10 \%$ thick PML is introduced to the fiber since the chances of the complexity of fabrications more to a value below this and the value above this introduces a bulky fiber. Zeno is chosen for the material because of several advantageous characteristics like high optical stability.

The light propagation of the waveguide happens in the core region. Hence, the terahertz radiation is narrowed to the core region. The waveguide is divided into elements that are used to evaluate the various guiding characteristics for better performance. To have polarization preserving application it is important to have high birefringence. Birefringence is defined as the difference between the refractive index of the polarization modes. It depends on the width of the rectangular-shaped air hole. With varying core lengths, the increase in core length at a particular width increases the birefringence because of an increase in refractive index difference. The best-acquired result is that the core length is taken as $300 \mu \mathrm{m}$ for higher birefringence and low material loss. It is also observed that the birefringence increases with an increase in operating frequency. A birefringence of 0.075 is obtained.

One of the key characteristics for effective terahertz propagation is to have a low effective material loss (EML). It occurs because the background material absorbs some of the light propagating through the waveguide. It depends on bulk material loss, the permittivity of air, permeability of free space, and effective refractive index of the material. The EML increases with an increase in operating frequency. This is because the light with higher frequency travels with more ease in the solid core. The EML result obtained for these conditions is $0.045 \mathrm{~cm}-1$.

Another kind of loss that is unavoidable in porous core microstructure fiber is confinement loss. This occurs due to the restriction of light in the core region. It depends on factors like operating frequency and velocity of light. The confinement loss decreases with higher 
frequency because it confines the core tightly. Further, if the width of the rectangular air holes increases, the fiber becomes highly porous and the light shows the tendency to leave the region. The best-observed result is that the obtained confinement loss is $7.544 \times 10-4 \mathrm{~cm}-1$ at 1.2 THz.

The next important factor is the power fraction, this ensures that the light is propagated through core air holes. This is important for effective transmission. It is observed that $47 \%$ of the total power transmits through the core air holes.

Bending loss occurs due to the power loss in bending of the optical fiber. It depends on factors like bending radius and effective area of guided-mode light where the light wave propagates. It is observed that the loss is lesser with increased frequency.

The waveguide dispersion indicates the amount of energy dispersed throughout the optical waveguide. It takes care of the geometry of the waveguide. It is calculated with the help of angular frequency, the velocity of light, and the effective refractive index of the guided wave. The dispersion for this fiber is $\pm 0.17 \mathrm{ps} / \mathrm{THz} / \mathrm{cm}$.

This type of porous core microstructure has been numerically proved to be the best method available so far. Over the years, several authors have worked on this area and have provided variations with improved characteristics. In the year 2016, some designs were proposed with numerical results varying between a birefringence of 0.03-0.05, EML of 0.06$0.08 \mathrm{~cm}-1$, dispersion variation of $0.2-1 \mathrm{ps} / \mathrm{THz} / \mathrm{cm}$, and power fraction of $30-50 \%$. This method has a better numerical value than almost all of the proposed results in the literature. Even though it is slightly difficult to fabricate the hexagonal type air hole cladding region due to its increased complexity, it can be done by using a technique called the Extrusion technique.

\subsection{SQUARE TYPE MICROSTRUCTURE FIBER}

To improvise the characteristics of the microstructure fiber, a square-shaped lattice whose air holes are circular is selected for the core and cladding area. This is done to ease the process of fabrication of the fiber. In the core region, the diameter of the core air holes is not constant. This is to ensure that the core porosity is varied. Further, the air holes in the cladding region are arranged in uniform rows and columns. Air Filling Fraction (AFF) is the ratio of the diameter of the air holes in the cladding area and the space between the adjacent air holes in uniform spacing. The AFF for the cladding region is taken as 0.97 and it is not varied. This square-type microstructure fiber also uses a Perfectly Matched Layer (PML). The PML is used to absorb and stop electromagnetic waves from reaching the surface. PML is situated outside the outer cladding region. The thickness of PML is taken to be $10 \%$ of the total diameter of the fiber and 
this value remains constant throughout. To get adequate results it is important to make use of good material. It needs to produce a low refractive index, low absorption loss, good transparency, and exceptional optical stability after exposure to the environment. Some of the materials that hold good to required conditions are Topas, Teflon, Silica, etc.

This Photonic Crystal Fiber (PCF) has several properties and they are analyzed. As light passes through the PCF several losses occur. One of them is Effective material loss or absorption loss. This can be reduced by variation of core porosity which is necessary because increased core porosity leads to a decrease in material loss. The EML result obtained for these conditions is $0.06 \mathrm{~cm}-1$. When the light propagating in the cladding region is trapped, it leads to confinement loss. This can be decreased by increasing the operating frequency in the core region by keeping the core porosity at a constant value. The obtained confinement loss for these conditions is $8.2 \times 10-4$. The above-mentioned factors are also numerically analyzed and verified. The dispersion variation for this model of microstructure fiber is $0.85 \pm 0.12 \mathrm{ps} / \mathrm{THz} / \mathrm{cm}$. This is one of the designs that were proposed in 2016 for improved waveguide characteristics of microstructure fiber.

\subsection{DESIGN AND ANALYSIS OF FOLDED-SHAPED CLADDING POROUS FOR APPLICATION OF OPTICAL SENSORS.}

A design of a different shaped cladding porous is offered and analyzed for sensing applications. It has circular air holes and this design type of photonic crystal fiber is also termed FPPCF. Due to identical properties of the PCF like linearity and a good amount of effective area, it contributes to high communications in the fiber and thus enhances the application of optical sensors. This proposed structure provides high sensitivity which is required for sensing application. The material best suited for this design is pure silica.

In the FP-PCF design, the porous microstructure fiber is filled with aqueous analyst ethanol. The core area of the fiber is surrounded by five-layered circular air holes which act as the cladding area for the fiber. The core region has two layers of air holes and the core radius is uniform. Due to several air holes in the cladding region and the presence of a chemical in the core region, the refractive index in the core region is greater than that of the cladding region. Hence, the mechanism of Total Internal Reflection is applied for FP-PCF for the propagation of light. Anisotropic PML is used to reduce the absorption of electromagnetic waves on the surface of the structure. Like in previous designs, the PML of this design is also $10 \%$ of the cladding region in total. 
To ensure the working of FP-PCF as a sensor it is important to analyze several factors like sensitivity, non-linearity, effective area, and confinement loss. These factors are simulated using software with the FEM mesh generation technique. For efficient results, full vectorial FEM is used. It's a strong numerical method. The waveguide structures of this PCF are divided into uniform spaces and the result is computed. For simulation of the PCF, the structure is divided into thousands of elements, and mesh generation is applied to it. This also consists of PCF parameters like an electric field. The result acquired is of complex numbers. The imaginary part of the result gives the confinement loss whereas the real part produces the effective mode index. Confinement loss occurs due to the penetration of power in the cladding area of the fiber. The effective area is calculated with the help of an electric field. The non-linearity of the PCF is calculated using the effective area and nonlinear coefficient of the material used in the PCF microstructure fiber. For the application of sensing, the relative sensitivity coefficient is calculated.

The Refractive index of the material and wavelength of the function are codependent. An increase in the wavelength leads to a linear decrease of the effective refractive index. The confinement loss of this FP-PCF is less because the light is tightly packed in the core region. This also means that the sensitivity is high and the fiber characteristics are as per requirement.

To improvise this structure, the PML depth is varied in increments of 5\% to check for the variation in confinement loss. The confinement loss is low for low PML depth. After performing the computation for varying PML depths, it is observed that the sensitivity is not affected by the change in PML. It remains constant. To increase the sensitivity of the PCF the cladding area is increased up to $5 \%$ by modifying the air hole characteristics. Higher sensitivity means better working of the PCF. For 5\% cladding area with 10\% PML, the sensitivity is obtained as $62.98 \%$. The confinement loss was found to be $1.38 \times 10-7 \mathrm{~dB} / \mathrm{m}$. To further increase the sensitivity of the material the core region area is increased to $5 \%$ which results in an increased sensitivity of $71.62 \%$. This however also leads to an increase in confinement loss to about 5.47 $\times 10-3 \mathrm{~dB} / \mathrm{m}$. Hence, this proposed change leads to a conflict between higher sensitivity and higher confinement loss. For high data transmission, larger effective area of PCF is required.

\section{FINITE ELEMENT METHOD SIMULATION OF GUIDED WAVE PHENOMENA AT TERAHERTZ FREQUENCIES}

Terahertz time-domain spectroscopy and imaging are essential and well-resolved techniques for biomedical imaging and trace gas detection of semiconductors. Experiments 
Terahertz time-domain spectroscopy. Spectroscopy and biomedical imaging in the Terahertz time-domain employ optical rectifications in nonlinear optical crystals.

Most devices use pulses with a broad wavelength and time-domain (100ghz-30 THz) [14] bandwidth in experiments. The development of these THz broadband pulses. The experiment consists of a prism, a mirror that focuses on near-infrared radiation and sees it. It takes a wide area of space. But it uses low depression, attenuation loss, to create optical waveguides. The FEM approach can be used for loss, depression, frequency, and time in the optical waveguide. But both theoretically and technically, this experiment should be performed. On the terahertz element, we can implement a terahertz waveguide.

The FEM model relies primarily on the concept of geometry, such as physical properties, modeling method, and execution work. Physical properties depend on son dielectric constant, RI, modeling method of magnetic permeability mainly sees continuous process primarily on dimensional analysis such as $2 \mathrm{D}$ structures such as triangular and 3D structures such as tetrahedral, creating mesh nodes and forming PDE simulation and execution are the related software-based that can solve PDE to find EM field in a specific location. For instance, electromagnet phenomena of the mesh element type are modeled by parameters such as constants that can be easily analyzed and these equations can be used in most problems except in parallel geometric shapes and can be solved in MATLAB.

The experimental method or waveguide is coupled to less than $1 \%$ beam, but using FEM, it is efficient to establish FEM using dual wire of terahertz couples. For the experimental model of ohmic waveguide loss, the coupling wire has a diameter and boundary state, surface impedance but perpendicular to the direction with a distance of 500 micrometers and a $\mathrm{k}$ vector with an incidence angle of 45 degrees [15].

Discretization into degrees of freedom in the realm of 3D simulation. A large number of mesh components is due to the density of the mesh. GMRES and SSOR can solve the solution. The multigrid approach utilizes a mesh hierarchy of varying densities. The software algorithm reduces solution time [18-19].

\subsection{TERAHERTZ SIGNAL IN BIOSENSING}

Terahertz signal is widely used in biosensing for the location of DNA, protein, and other biomedical materials because it has few advantages as it is a nondestructive, label-free, and non-contact manner of terahertz range, and they are analyzed and simulated by FEM even in $\mathrm{X}$-rays and UV rays allow terahertz ranges in biomedical materials. The targeted virus can be investigated further by the terahertz signal. Terahertz waveguides are used in high and targeted 
viruses by terahertz spectroscopy with desirable frequencies. Then these biosamples are further investigated by optical formalities by finite difference time domain (FDTD) and FEM are complexed refractive index, absorbed characteristics. In this way, terahertz signals are used in biosensors.

The virus sample is put in a chip with an antenna containing terahertz radiation. Nanoantenna is used in the experiment. The virus builds thin film with appearance. It forms resonant frequency, time domain, and other characteristics and further can be used for simulation in finite difference time domain method (FDTD) cladding and perfectly matched layer (PML) is done in the simulation. Moreover, this method uses terahertz signal patterns with antenna and spectral changes indicate virus detention

\subsection{TERAHERTZ SIGNAL IN LIQUID METAL COMPONENTS}

There are many applications in terahertz signals.

Terahertz signals are used for communications purposes in research activities. Solid metals have a consistent of only 100 Gigahertz and they are called mono-functional devices. In further development liquid metals like Galinstan, Gallium-Indium tin alloy having high conductivity and low toxicity. An electrolytic solution consisting of $2 \mathrm{M} \mathrm{NaOH}$ in water having an absorption coefficient through the terahertz range.

We can use a terahertz signal as coupled waveguide filter is added in a spectral selection of multiplexed terahertz signal. We can couple from top to bottom waveguide. The filter capillary is immersed in liquid electrolyte with terahertz beam path with DC the coupling in two waveguides in exclusively for wall and simulation is done by FEM for coupling of two waveguide and transmissions arises.

New designs can be constructed by the terahertz signals processing component. We can perform filtering in both 2D and 3D by FEM and by time-domain spectroscopy.

\subsection{TERAHERTZ SIGNAL IN PETROL ADULTERATION}

Another application where FEM is used is petrol adulteration. Sensor crystal fibers are used and analyzed by terahertz signals. FEM simulation can relate sensitivity and confinement loss. Photonic crystal fibers are recently used in the device because of design flexibility. Numerical analysis for petrol adulteration can be simulated by software with FEM and perfectly matched layer (PML) boundary conditions are used for cladding purposes having $10 \%$ thickness in sensor structure. Several waveguides are proposed for petrol adulteration like hollow-core waveguides and photonic crystal. Alternatives and changes in liquid are determined by changes in 
liquid in the terahertz region and numerically analyzed and solved. FEM is used to demonstrate to the core and simulations are obtained.

The photonic band is now used in all places as it has advanced application and material used is silicon photonics and other materials also can be used in finding other tremendous methods to find a photonic gap in optical source

Photonic devices are both active and passive devices and have real-life application and when the crystal produces bandwidth and it is numerically analyzed by FEM

\subsection{PHOTONIC CONDUCTIVE TERAHERTZ ANTENNA WITH RADIAL SYMMETRY}

Terahertz metal wire waveguide experiment with low coupling efficiency. The photoconductive antenna is used in an antenna with cylindrical symmetry with two dc biased electrodes for acceleration. Terahertz antenna design is non-cost effective. We can use FEM design for terahertz antenna and waveguide. Bandwidth in a photonic band is a periodic structure with light wavelength and refractive index constant and light cannot travel in all directions.

Electromagnetic wave propagation is one of the difficult methods to simulate wave propagation. It can be simulated by the mesh element size. FEM computation and communication can be done on a personal computer. The finite difference time domain method (FDTD) is another method used to find the solution. FEM model is increased in memory for solving model. FEM model to solve electromagnetic device. FEM software for electromagnetic simulation can only solve important problems like wave optics, wave propagation in electromagnetics. FEM simulation results can give values with two wires. It gives a 0.1 terahertz wave with $\mathrm{X}$ component of the electric field. FEM simulation in antenna device in fabrication for radial antenna, durability is increased for polarization of light.

FEM simulation can be used for terahertz beam and simple calculation is done by FEM.

\subsection{FINITE ELEMENT METHOD SIMULATION IN RADIAL ANTENNA}

It is very easy to design a radial antenna ideally, but practically it's very difficult to modify the dielectric constant. We compare the real and ideal antenna design using FEM, in the YZ plane for disc showed with radius.

FEM is used for coupling terahertz signal and antenna source. FEM simulation with photonic crystal with the transmission of radiation through the antenna. Recently it is used in research and experimental activities. 


\section{FINITE SIMULATION RESULTS}

After the experiment wave shatters and a radial waveguide mode is created, we can plot that along the Z-axis in the $\mathrm{x}$ electrical field part. With $0.1 \mathrm{THz}$, a sine wave is incited with both forward and backward oscillations and attenuation is achieved. Owing to differences in mesh densities, there may be fluctuations. The outcome is created by wave demonstration under the limitations of boundary conditions. Where the wire length can be the Z-axis and the simulation domains can be XY. It is difficult to decide whether excitation occurs in a single mode. We can get an input wave in a cylinder that acts as a perfect example for the FEM simulation model by integrating time average power over the cylinder circular base field. The efficiency generated is 0.42 percent by calculation. In antenna model research and manufacturing and simulation, efficiency varies concerning wavelength, wire separation, and polarization of waveguide FEM may be used.

\section{CONCLUSION}

In this paper, we have talked about the importance of finite element analysis in optical waveguides and terahertz frequencies. FEM has seen a vast development over the years. This paper has reviewed the fundamentals of FEM like the theoretical framework, nodal and edge elements, and the Perfectly Matched Layers. The FEM techniques in Beam Propagation and Waveguide discontinuity were discussed. Finite element analysis is the most widely used algorithm because of the simpler modeling of complex geometric structures, the simplicity of the computer, precision, time-dependent simulations. In the first section, we have introduced numerical approximation using FEM, which is applied in fluid dynamics, heat transfer, and wave propagation. For today's generation use of software design, computation, solving in the approximation is essential. FEM is a very important method for these applications.

In the microstructure fiber, hexagonal type air hole was used in the cladding region and rectangular-shaped air holes in the core region. This was proved to be advantageous in improving the guided characteristics with the help of FEM. Numerical findings indicate that there is a strong birefringence of 0.075 , an efficient loss of $0.045 \mathrm{~cm}-1$, a high-power fraction of 47 percent, and a low dispersion of $\pm 0.17 \mathrm{ps} / \mathrm{THz} / \mathrm{cm}$ inside the structure being proposed. Furthermore, in the cladding and core region, we implemented a square lattice and analyzed the parameters using number-cal technology. We have obtained the EML as $0.06 \mathrm{~cm}-1$, an $8.2 \mathrm{x}$ $10-4$ load of containment, and a $0.85 \pm 0.12 / \mathrm{THz} / \mathrm{cm}$ very low dispersion. The guiding characteristics of FP-PCF were analyzed for the increased effective area and high sensitivity due to 
the increase in the application of PCF in the area of sensing. Other factors like nonlinearity and confinement loss were also estimated.

Terahertz optical waveguide helps us to investigate AI virus with terahertz spectroscopy with Nanoantenna with single resonance optimization. Moreover, detection of the virus. As it is very important as it may cause pandemic FEM, FDTD helps us to find more about the virus with high accuracy

We perform FEM in both 2D and 3D and liquid metal can be used as an electrolyte as it has high conductivity. Petrol adulteration is an important application and FEM can be used for analysis. Terahertz waveguide shows high numerical aperture and it is used in real application because petrol can be mixed with alcohol and other substance. We can insert sensors activities and find the relative sensitivity and accuracy through FEM

FEM is used in research, CAD lab, modeling, imaging, and time spectroscopy as an important method as it saves computational time and money. It also facilitates the development of the design. Recent advancements in programming will lead to less computation time and easier manners to obtain accurate numerical solutions. For research activities, FEM is now a globally important subject.

\section{References}

[1] J. F. Lee, D. K. Sun and Z. J. Cendes, "Tangential vector finite elements for electromagnetic field computation," in IEEE Transactions on Magnetics, vol. 27, no. 5, pp. 40324035, Sept. 1991, doi: 10.1109/20.104986.

[2] F. L. Teixeira and W. C. Chew, "General closed-form PML constitutive tensors to match arbitrary bianisotropic and dispersive linear media," in IEEE Microwave and Guided Wave Letters, vol. 8, no. 6, pp. 223-225, June 1998, doi: 10.1109/75.678571.

[3] K. Saitoh and M. Koshiba, "Full-vectorial finite element beam propagation method with perfectly matched layers for anisotropic optical waveguides," in Journal of Lightwave Technology, vol. 19, no. 3, pp. 405-413, March 2001, doi: 10.1109/50.918895.

[4] Y. Tsuji and M. Koshiba, "Finite element method using port truncation by perfectly matched layer boundary conditions for optical waveguide discontinuity problems," in Journal of Lightwave Technology, vol. 20, no. 3, pp. 463-468, March 2002, doi: $10.1109 / 50.988995$.

[5] Attila Mekis, J. C. Chen, I. Kurland, Shanhui Fan, Pierre R. Villeneuve, and J. D. Joannopoulos "High Transmission through Sharp Bends in Photonic Crystal Waveguides" Phys. Rev. Lett. 77, 3787 - Published 28 October 1996. 
[6] M.A. Habib, M.S. Anower, M.R. Hasan, Highly birefringent and low effective material loss microstructure fiber for the wave guidance, Opt. Commun. 423 (2018) 140-144.

[7] R. Islam, M. S. Habib, G. K. M. Hasanuzzaman, R. Ahmad, S. Rana and S. F. Kaijage, "Extremely High-Birefringent Asymmetric Slotted-Core Photonic Crystal Fiber in THz Regime," in IEEE Photonics Technology Letters, vol. 27, no. 21, pp. 2222-2225, 1 Nov.1, 2015, doi: 10.1109/LPT.2015.2457673.

[8] M.R. Hasan, M.S. Anower, M.I. Hasan, S.M.A. Razzak, Polarization maintaining low-loss slotted core kagome lattice the fiber, IEEE Photon. Technol. Lett. 28 (16) (2016) 17511754.

[9] M.A. Habib, M.S. Anower, Design and numerical analysis of highly birefringent single mode fiber in the regime, Opt. Fiber Tech. 47 (2019) 197-203.

[10] R.G. Hunsperger, Optical waveguide modes. in Integrated Optics. 3rd edn., ed. by R.G. Hunsperger (Springer, New York, 2009), pp. 17-31.

[11] J. Jin, The Finite Element Method in Electromagnetics, 2nd edn. (Wiley, NY, 2015), pp. 233-633. ISBN13 9780471438182

[12] D. Dai, Z. Sheng, JOSA B $24(11), \quad 2853-2859 \quad$ (2007). https://doi.org/10.1364/JOSAB.24.002853.

[13] L.E.G. Castillo, A.J.R. Genoves, I.G. Revuelto, IEEE Trans. Magn. 28, 5 (2002). https://doi.org/10.1109/TMAG.2002.803577

[14] K. Wang and D. M. Mittleman, BGuided propagation of terahertz pulses on metal wires,[ J. Opt. Soc. Amer. B, vol. 22, pp. 2001-2007, 2005.

[15] Y. Zhu and A. Cangellaris, Multigrid Finite Element Methods for Electromagnetic Field Modeling, ser. The IEEE Press Series on Electromagnetic Wave Theory, D. G. Dudley, Ed. Hoboken, NJ: Wiley, Inc., 2006.

[16] K. Wang and D. M. Mittleman, BMetal wires for terahertz waveguiding, [ Nature, vol. 432, p. $376,2004$.

[17] Z. Jian and D. M. Mittleman, BOut-of-plane dispersion and homogenization in photonic crystal slabs,[ Appl. Phys. Lett., vol. 87, p. 191 113, 2005 T. Prasad et al., BThe superprism effect in a metal-clad terahertz photonic crystal slab,[ Opt. Lett., vol. 32, pp. 683-685, 2007.

[18] Paul, B.K. and Ahmed, K., 2020. Analysis of terahertz waveguide properties of Q-PCF based on FEM scheme. Optical Materials, 100, p.109634.

[19] Tukmakova, A.S., Asach, A.V., Novotelnova, A.V., Tkhorzhevskiy, I.L., Kablukova, N.S., Demchenko, P.S., Zaitsev, A.D. and Khodzitsky, M.K., 2020. FEM Simulation of 
J. Sustain. Wireless Syst., vol. 03, no. 2, pp. 68-86

https://doi.org/10.36548/jsws.2021.2.002

$\mathrm{THz}$ Detector Based on $\mathrm{Sb}$ and Bi88Sb12 Thermoelectric Thin Films. Applied Sciences, 10(6), p.1929.

[20] Morimoto, K., Iguchi, A. and Tsuji, Y., 2020. Efficient topology optimization of optical waveguide using finite element method based on slowly varying envelope approximation. Optik, p.165951.

[21] Agrawal, A., 2019. Applications of Finite Element Method: Modelling of multilayer stacks/anti-reflection coatings.

[22] Liu, N., Cai, G. and Liu, Q.H., 2016, August. The efficient finite element method with impedance transmission boundary condition for computing optical waveguide modes. In 2016 URSI Asia-Pacific Radio Science Conference (URSI AP-RASC) (pp. 1-2). IEEE.

[23] Habib, Md \& Anower, Md Shamim. Square Porous Core Microstructure Fiber for Low Loss Terahertz Applications (2019). 126. 690. 10.21883/OS.2019.05.47671.335-18.

[24] Bikash Kumar Paul, Kawsar Ahmed, Sayed Asaduzzaman, Md. Shadidul Islam, Folded cladding porous shaped photonic crystal fiber with high sensitivity in optical sensing applications: Design and analysis. https://doi.org/10.1016/j.sbsr.2016.11.005.

[25] Sivaganesan, D. "IMPROVISATION OF MESH NETWORK WITH WIDEBAND CODE DIVISION MULTIPLE ACCESS." Journal: IRO Journal on Sustainable Wireless Systems September 2019, no. 03 (2019): 198-205.

[26] Raj, Jennifer S. "Machine Learning Implementation in Cognitive Radio Networks with Game-Theory Technique." Journal: IRO Journal on Sustainable Wireless Systems June 2020, no. 2 (2020): 68-75.

[27] Smys, S., Haoxiang Wang, and Abul Basar. "5G Network Simulation in Smart Cities using Neural Network Algorithm." Journal of Artificial Intelligence 3, no. 01 (2021): 43-52.

[28] Bashar, Abul. "Sensor Cloud Based Architecture with Efficient Data Computation and Security Implantation for Internet of Things Application." Journal of ISMAC 2, no. 02 (2020): 96-105

[29] Bhalaji, N. "Reliable Data Transmission with Heightened Confidentiality and Integrity in IOT Empowered Mobile Networks." Journal of ISMAC 2, no. 02 (2020): 106-117.

[30] Adam, Edriss Eisa Babikir. "Evaluation of Fingerprint Liveness Detection by Machine Learning Approach-A Systematic View." Journal of ISMAC 3, no. 01 (2021): 16-30.

[31] Duraipandian, M. "Ranked k-NN Crowdsourced Model for Cloud Internet of Things (CIoT)." Journal of ISMAC 2, no. 03 (2020): 173-180. 